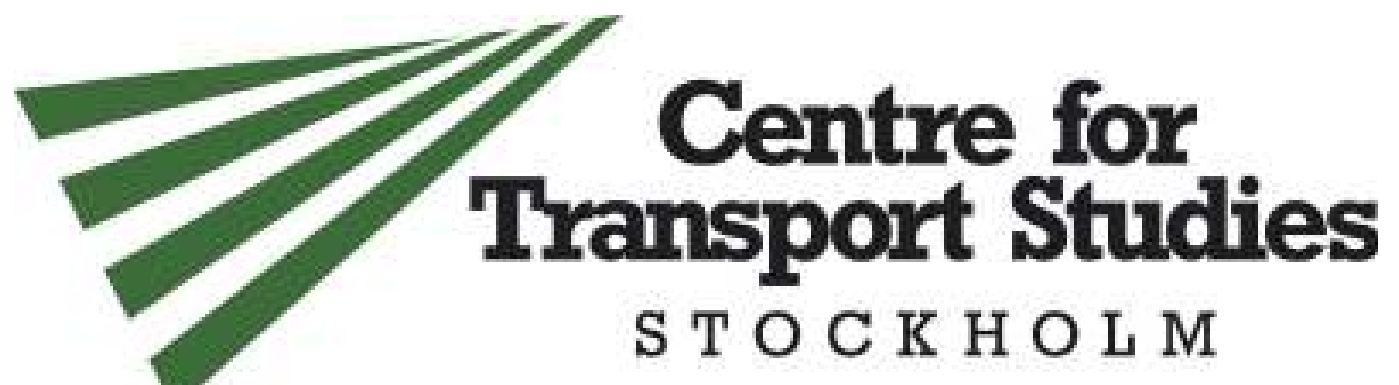

\title{
Marginal Railway Track Renewal Costs: A Survival Data Approach
}

\author{
Mats Andersson - VTI \\ Gunilla Björklund - VTI
}

CTS Working Paper 2012:29

\begin{abstract}
In this paper, renewal costs for railway tracks are investigated using survival analysis. The purpose is to derive the effect from increased traffic volumes on rail renewal cycle lengths and to calculate associated marginal costs. A flow sample of censored data containing almost 1300 observations on the Swedish main railway network is used. We specify Weibull accelerated failure time regression models, and estimate deterioration elasticities for total tonnage as well as for passenger and freight tonnages separately. Marginal costs are calculated as a change in present values of renewal costs from premature renewal following increased traffic volumes. The marginal cost for aggregate freight and passenger tonnage is estimated to approximately SEK 0.002 per gross ton kilometre.
\end{abstract}

Keywords: Railway; Renewal; Survival analysis; Marginal costs

JEL Codes: C41; H54; L92; R48

Centre for Transport Studies SE-100 44 Stockholm Sweden www.cts. kth.se 



\title{
Marginal Railway Track Renewal Costs:
}

\author{
A Survival Data Approach
}

Mats Andersson ${ }^{\mathrm{a}}$, Gunilla Björklund ${ }^{\mathrm{a}, *}$

${ }^{a}$ Swedish National Road and Transport Research Institute (VTI), and Centre for Transport Studies, Box 55685, SE-102 15 Stockholm, Sweden 


\begin{abstract}
In this paper, renewal costs for railway tracks are investigated using survival analysis. The purpose is to derive the effect from increased traffic volumes on rail renewal cycle lengths and to calculate associated marginal costs. A flow sample of censored data containing almost 1300 observations on the Swedish main railway network is used. We specify Weibull accelerated failure time regression models, and estimate deterioration elasticities for total tonnage as well as for passenger and freight tonnages separately. Marginal costs are calculated as a change in present values of renewal costs from premature renewal following increased traffic volumes. The marginal cost for aggregate freight and passenger tonnage is estimated to approximately SEK 0.002 per gross ton kilometre.
\end{abstract}

Keywords: Railway; Renewal; Survival analysis; Marginal costs

JEL Classification: C41; H54; L92; R48 


\section{Introduction}

Rail infrastructure managers face the problem of track wear and tear in their daily business. This is caused by operating train services as well as non-traffic factors such as location and climate. The wear and tear process can be managed by undertaking maintenance activities, but eventually a more substantial renewal activity is necessary. The wear and tear question should therefore be seen as a joint maintenance and renewal problem. Pricing infrastructure wear and tear is of great importance from an efficiency standpoint. Over the last decade, research on the subject has gradually increased for all modes of transport, both in Sweden and internationally (Andersson, 2008; Andersson, 2011; Bruzelius, 2004; Nash, 2003; Nash and Matthews, 2005; Nash and Sansom, 2001; Thomas et al., 2003; Wheat and Smith, 2008). Sweden has a long tradition of marginal cost pricing in the transport sector, but to date, railway infrastructure wear and tear fees have excluded costs for rail renewal. This issue has drawn some attention regarding the lack of empirical evidence on the size of a pricing relevant rail renewal cost component (Nash, 2005).

The fee for railway infrastructure wear and tear in Sweden is based on econometric analyses of infrastructure operation and maintenance costs by Johansson and Nilsson (2004) and Andersson (2006). Johansson and Nilsson (2004) use cost data from the mid 1990's, but detailed information on renewals was not available at the time of their analysis. Andersson (2006, 2008) extends their analysis with data from 1999 to 2002 by analysing an aggregate of maintenance and renewal costs using initially pooled ordinary least squares (POLS) and later fixed effects (FE) models. He finds a higher cost elasticity with respect to output than Johansson and Nilsson (2004) and increased marginal costs in the POLS approach, but a lower cost elasticity in the FE approach. The POLS approach was rejected and furthermore, the cost function approach to identify the marginal cost of the aggregate of maintenance and renewal is questioned. As rail renewals have long life cycles (are rare events), the lack of 
comprehensive time-series data questions the adequacy of applying traditional regression analysis to the renewal problem. An alternative econometric approach to model rail renewal costs is presented in Andersson et al. (2012). They use models for censored and truncated data to model the renewal cost problem. The results are promising in terms of elasticity and marginal cost estimates. The recommended model is a two-part model that deals with the renewal problem in two stages. Stage one models the decision to renew or not using a probit model and stage two deals with the size of the renewal using a truncated regression model. In contrast to previous applications of econometric techniques, we suggest a different approach in this paper using an analytical expression for the marginal rail renewal cost and survival analysis of track segments as input to the marginal cost calculation.

In the present paper, almost 1300 railway track segment observations are used to analyse rail life in relation to freight and passenger traffic in Sweden. Weibull survival functions in accelerated failure time form are estimated using rail life as dependent variable while traffic and other infrastructure variables are used as covariates.

The main findings are that the estimated models give a good fit of the data. We find strong positive duration dependence, with a more than proportional increase in renewal probability over time with respect to accumulated traffic. This supports the choice of the Weibull model for this data. The weighted average marginal cost for aggregate freight and passenger tonnage are estimated to approximately SEK 0.002 per gross ton kilometre ${ }^{1}$

The outline of the paper is as follows. In section 2, we describe the modelling approach followed by a data review in section 3 . The model results are presented in section 4 . Marginal costs are calculated in section 5 while section 6 concludes.

\footnotetext{
${ }^{1}$ The exchange rate from Swedish Kronor (SEK) to Euro (EUR) is SEK 8.63/EUR and from Swedish Kronor to US Dollar (USD) is SEK 6.61/USD (December 6, 2012).
} 


\section{The modelling approach}

\section{$\underline{2.1 \text { Modelling rail life }}$}

Survival data are used in a number of research disciplines. In medicine, it can be the case of time elapsed between a treatment and a specific health state. In labour economics, it can be the time spell of a person being unemployed or in engineering, the time until a component fails to perform its intended function.

In this paper, the expected life of railway track segments is analysed using parametric survival models. The general theory and concepts of survival analysis and model estimation is well developed, e.g. Kiefer (1988), Lancaster (1990) or Klein and Moeschberger (2002). We follow the terminology of Klein and Moeschberger (2002) in the presentation of the underlying theory of the survival analysis.

Let $X$ be a nonnegative random variable, representing the time in years between two railway track segment renewals i.e. rail life. There are a few different ways of characterising the distribution of $X$ and if we know any of these, the others can be identified. First, the distribution of $X$ can be represented by a survival function. The survival function $S$ states the probability $P$ of an individual track segment surviving beyond time $x$.

$$
S(x)=P(X>x)
$$

The survival function is the complement to the cumulative distribution function $F$, $S(x)=1-F(x)$, where $F(x)=P(X \leq x)$. The probability density function $f$ gives the unconditional probability of a track segment being renewed in time $x, f(x)=-\frac{d S(x)}{d x}$. 
Second, the probability that a track segment of age $x$ will be renewed instantaneously after $x(x+\Delta x)$ is represented by the hazard rate $h$. The hazard function for a continuous variable is defined by

$$
h(x)=\lim _{\Delta x \rightarrow 0} \frac{P[x \leq X<x+\Delta x \mid X \geq x]}{\Delta x}=\frac{f(x)}{S(x)}=\frac{-d \ln [S(x)]}{d x} .
$$

The cumulative (or integrated) hazard function $H$ is defined as

$$
H(x)=\int_{0}^{x} h(u) d u=-\ln [S(x)] .
$$

The probability density function $f(x)$ can also be expressed using the hazard function and the cumulative hazard as in (4).

$$
f(x)=h(x) \exp [-H(x)]
$$

The Weibull model is used in the analyses, which is a popular parametric model for engineering survival data. The model has a survival function $S(x)=\exp \left[-\lambda x^{\alpha}\right]$ for $x>0$. $\lambda>0$ and $\alpha>0$ are known as the scale and shape parameters respectively. The hazard rate has the form of $h(x)=\lambda \alpha x^{\alpha-1}$ and the cumulative hazard $H(x)=\lambda x^{\alpha}$. The probability density function and cumulative distribution function are expressed as $f(x)=\lambda \alpha x^{\alpha-1} \exp \left[-\lambda x^{\alpha}\right]$ and $F(x)=1-\exp \left[-\lambda x^{\alpha}\right]$ respectively. Finally, $\mu$ is the expected value of the renewal interval, $E(X)=\mu=\frac{\Gamma(1+1 / \alpha)}{\lambda^{1 / \alpha}}$, where $\Gamma$ is the Gamma function. 


\subsection{An analytical approach to marginal renewal costs}

The theory behind the approach that we use is developed within a context of structural road repair, but is applicable to any transport mode (Link and Nilsson, 2005). The initial presentation is based on a deterministic model, which later is extended to include stochastic parts. The baseline is that the time span between two renewals is decided by accumulated traffic on a specific segment. Newbery (1988) assumed that the amount of traffic that a road could handle is decided upon in the design phase and hence will affect construction costs. Assuming the prediction of traffic is correct and the sole contributor to deterioration, he introduced the so called Fundamental theorem, i.e. short run marginal cost of road damage equals average cost. Newbery's theory is extended in Lindberg (2002) who formulates a more general expression for calculation of marginal costs, which we use for the railway track analysis. We will therefore discuss Lindberg's model within a railway context. The life time of a track segment is defined as

$$
X=\left[\frac{\Theta(q)}{q}\right] e^{-m X}
$$

where $X$ is the renewal interval, $\Theta$ is accumulated tonnage that the track can accommodate between two renewals, $q$ is annual tonnage and $m$ is non-traffic related deterioration. The concept of Lindberg's approach is that the total tonnage is a function of actual annual traffic, rather than being a constant that can be predicted in advance as Newbery assumes.

Furthermore, non-traffic deterioration can shorten the renewal interval in the form $e^{-m X}$.

The model assumes that the track has an initial quality of $\mathrm{Q}^{\mathrm{H}}$ (Figure 1). Traffic volumes reduce this quality over time and a renewal of the track is justified at $X^{*}$ with quality $\mathrm{Q}^{\mathrm{L}}$, when the initial quality level $\mathrm{Q}^{\mathrm{H}}$ is restored. Assuming constant traffic flows, this cycle is 
repeated into infinity with all future renewal intervals being of length $\bar{X}$. The deterioration of quality over time is associated with a railway track management cost, which can be discounted to any given reference year. The change in costs associated with a marginal increase in traffic $(\Delta q)$ in a specific year on a specific track segment is of main interest. Following Lindberg (2002), a negative association between $q$ and $X$ is expected, i.e. more traffic will shorten the renewal interval. The traffic increase at $\tilde{x}$ will affect the quality of the track and shorten the first renewal interval to $X$. Hence, renewal will take place at $X$ rather than $X^{*}$, and all subsequent renewals will be scheduled earlier than if the increase had not taken place. Discounting and comparing the two alternative cost streams in Figure 1 gives the marginal cost associated with the increase in traffic. This is of course a general presentation of the deterioration pattern that is affected by different aspects in the real world.

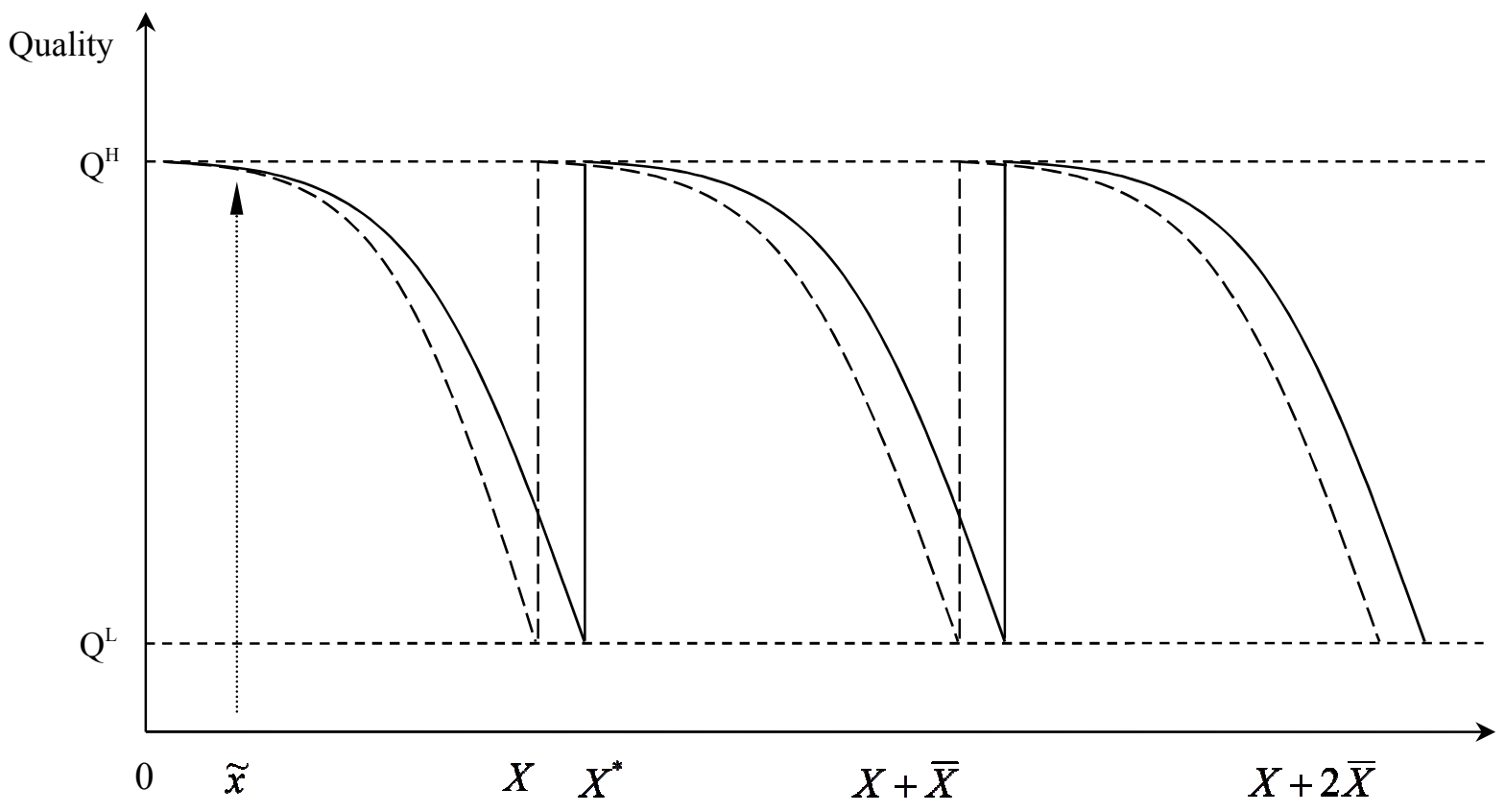

Time

Figure 1. Renewal intervals with and without a marginal increase in traffic at $\tilde{x}$ 
A schematic view of how the change in traffic affects traffic volumes and renewal intervals is given in Figure 2. In all time periods but one (the first renewal interval $X$ ), an observed traffic volume $q$ is assumed (as in $x$ in Figure 2).

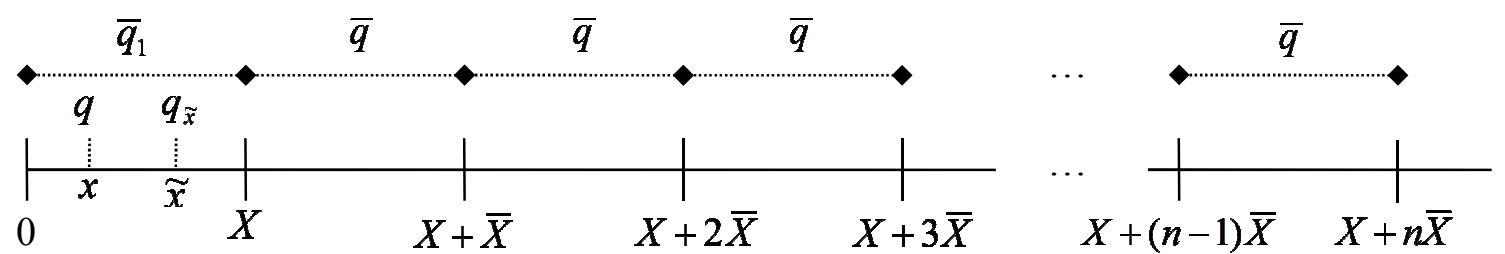

Figure 2. Traffic volumes and renewal intervals from a marginal increase at $\tilde{x}$

We further assume that the increase in traffic occurs in a specific time period, $\tilde{x}$, changing traffic volume from $q$ to $q_{\widetilde{x}}=q+\Delta q$. The average traffic volume in $X$ is then defined as $\bar{q}_{1}=\frac{\left(q(X-1)+q_{\widetilde{x}}\right)}{X}=q\left(1-\frac{1}{X}\right)+\frac{q_{\widetilde{x}}}{X}$. The traffic increase can be viewed as a shock to a system that returns to normal already in the next time period. This gives us marginal traffic change that can be associated with a cost change in later stages. After the renewal in $X$, traffic volumes of $q$ are used as a simplifying assumption in each time period giving constant average traffic flows $(\bar{q})$ and renewal intervals $(\bar{X})$ into infinity.

A track renewal comes at a cost $c$ expressed as SEK per track kilometre. The present value of an infinite series of renewals at $X$ with subsequent constant intervals $\bar{X}$ can be expressed as

$P V C_{X}=c\left(1+e^{-r \bar{X}}+e^{-r 2 \bar{X}}+\ldots+e^{-r n \bar{X}}\right)$, 
where $r$ is the social discount rate. When $n$ approaches infinity the present value of the renewal cost can be written as

$$
\lim _{n \rightarrow \infty} P V C_{X}=c \frac{1}{\left(1-e^{-r \bar{X}}\right)}
$$

A track segment that is observed in the first renewal interval at time $\tilde{x}$ will have $(X-\tilde{x})$ years remaining before the next renewal occurs. We define the remaining life time $\omega \equiv X-\tilde{x}$, which gives the present value of a track segment analysed in time $\tilde{x}$ as

$$
P V C_{\widetilde{x}}=c e^{-r \omega} \frac{1}{\left(1-e^{-r \bar{X}}\right)}
$$

The present value calculation then consists of two parts where the first is related to the current renewal interval $X$ and the second to all future (constant) intervals $\bar{X}$. As traffic affects the renewal interval, expression (8) is used to calculate the marginal cost, based on the change in present value from a change in traffic. $q_{\widetilde{x}}$ is observed annual traffic in $\widetilde{x}$ and we take the derivative of $P V C_{\widetilde{x}}$ with respect to $q_{\widetilde{x}}$. Following Haraldsson (2007),

$$
M C_{\widetilde{x}}=\frac{\partial P V C_{\widetilde{x}}}{\partial q_{\widetilde{x}}}=\frac{\partial P V C_{\widetilde{x}}}{\partial X} \frac{\partial X}{\partial q_{\widetilde{x}}}=-c r \frac{e^{-r \omega}}{\left(1-e^{-r \bar{X}}\right)} \frac{\partial X}{\partial q_{\widetilde{x}}} .
$$

Introducing the concept of a deterioration elasticity, $\varepsilon=\frac{\partial X}{\partial \bar{q}_{1}} \frac{\bar{q}_{1}}{X}$, as a measure of the change in the first renewal interval from a percentage change in traffic, we get expression (10) 
(Haraldsson, 2007), where $\bar{q}_{1}$ is the average annual traffic volume of the first renewal interval.

$$
\frac{\partial X}{\partial q_{\widetilde{x}}}=\frac{\partial X}{\partial \bar{q}_{1}} \frac{\partial \bar{q}_{1}}{\partial q_{\widetilde{x}}}=\left[\frac{\partial \bar{q}_{1}}{\partial q_{\widetilde{x}}}=\frac{1}{X}\right]=\frac{\varepsilon}{\bar{q}_{1}}
$$

(10) in (9) gives

$$
M C_{\widetilde{x}}=\frac{\partial P V C_{\widetilde{x}}}{\partial q_{\widetilde{x}}}=-c r \frac{e^{-r \omega}}{\left(1-e^{-r \bar{x}}\right)} \frac{\varepsilon}{\bar{q}_{1}}
$$

Haraldsson (2007) develops the theoretical foundation in Lindberg (2002) further to situations when renewal intervals are not deterministic, but follow some probability density $g$, positive for positive arguments.

$$
E\left[\frac{\partial P V C_{\widetilde{x}}}{\partial q_{\tilde{x}}}\right]=-c r \frac{\varepsilon}{\bar{q}_{1}} \int_{0}^{\infty} \frac{e^{-r \omega}}{\left(1-e^{-r \bar{X}}\right)} g(\omega) d \omega
$$

Given the survival function $S$ for the track life time, the density function for the remaining life time in a renewal process is $g(\omega)=\frac{S(\omega)}{\mu}$, where $\mu=E(X)$ as before (Lancaster, 1990). Assuming Weibull distributed lifetimes, $g(\omega)=\frac{e^{-\lambda \omega^{a}}}{\mu}$. This gives expression (13), the expected present value of the marginal cost with respect to tonnage, which has no closed form 
and must be solved with numerical integration ${ }^{2}$.

$$
E\left[\frac{\partial P V C_{\widetilde{x}}}{\partial q_{\tilde{x}}}\right]=-c r \frac{\varepsilon}{\bar{q}_{1}} \int_{0}^{\infty} \frac{e^{-r \omega}}{\left(1-e^{-r \bar{X}}\right)} \frac{e^{-\lambda \omega^{a}}}{\mu} d \omega=-\varepsilon \frac{c}{\bar{q}_{1} \mu} \frac{r}{\left(1-e^{-r \bar{X}}\right)} \int_{0}^{\infty} e^{-r \omega-\lambda \omega^{\alpha}} d \omega
$$

The deterioration elasticity $(\varepsilon)$ is estimated in the survival model with an accelerated failure time (AFT) Weibull error specification. The additional parameters to be derived from the survival analysis are the expected life time of a track segment $(\mu)$, and the Weibull scale $(\lambda)$ and shape $(\alpha)$ parameters. $c$ is the cost per kilometre for renewing a railway track, $\bar{q}_{1}$ is the average annual traffic volume of the first renewal interval and $r$ is the social discount rate.

\section{Railway data}

The infrastructure data at hand have been collected from the Swedish Rail Administration's (Banverket) ${ }^{3}$ track information system (BIS). BIS contains information about homogeneous track segments on the main lines in Sweden. Station areas, sidings, and freight marshalling yards are excluded from the analysis due to difficulties in allocating traffic volumes to such individual segments. Some segments are also excluded due to no information on traffic or age. Each included segment has information on which year the track was laid and some additional technical characteristics of the track as well as organisational identity.

Annual data between the years 1999 and 2009 are collected from BIS. Rail renewals between each of these years are identified through changes in the infrastructure information.

\footnotetext{
${ }^{2}$ Note that this is an approximation to a complete stochastic formulation of the marginal cost expression, in which $\bar{X}$ should also be a random variable as future renewal intervals are unknown and stochastic by nature. As $X$ also is stochastic, $\varepsilon$ is stochastic. This extension is however beyond the scope of this paper.

${ }^{3}$ The Swedish Rail Administration (Banverket) merged with the Swedish Road Administration (Vägverket) on April 1, 2010, and formed the Swedish Transport Administration (Trafikverket). All our data has been collected from Banverket, but henceforth we refer to Trafikverket as the provider of information as Banverket no longer exists.
} 
From the information on which year the track is laid, we derive an age variable for each observation. Any change during the study period results in an uncensored observation for the initial track that is replaced, i.e., where we observe the full length of the renewal cycle. For an observation with no changes registered, a censored observation is added with age as per the end of 2009.

The decision to renew a track segment is assumed to be taken when the track has reached a specific quality target. In real life, such a unique quality target is non-existent, but more likely the decision will be based on several information sources about the quality of the track. The overall objective though is always to minimise life cycle cost. When the discounted life cycle cost of operating and maintaining the old track in the future exceeds the discounted sum of the renewal cost and future track operation and maintenance costs, a renewal is justified. In our context, wear and tear over time from train passages will affect the cost of infrastructure operation and maintenance, and hence decide the optimal timing of a renewal.

Since no comprehensive traffic database exists in Sweden, a time-series of traffic data is created based on various sources of information. Traffic data for the period 1999-2002 is provided by Andersson (2006), who collected data from Swedish train operators. For the years 2007 to 2009 the traffic data is received from Banverket, and from 2003 to 2006, we interpolate traffic tonnage using traffic growth coefficients derived from access charge declarations by train operators to Banverket. This method gives an estimate of annual track segment traffic for the time window 1999-2009.

We started with a data set consisting of 1656 observations. After omitting track segments that were constructed during the time 1999-2009, and segments that did not have observed age or traffic data, the number of observations is reduced to 1272 , of which 1109 observations are censored (87.2 per cent). The mean age for the 1272 observations in the sample is 26 years at the end of year 2009, which includes both observed life times 
(uncensored observations) and current ages (censored observations). The great amount of censored observations implies that the predicted mean age for the track segments in the sample is probably much higher than 26 years. Sixty three per cent of main line track segments are single tracks and 82 per cent have continuously welded rails.

Table 1. Descriptive statistics of the uncensored observations in the data set

\begin{tabular}{lrrrr}
\hline Variable & Mean & Std. Dev. & Min & Max \\
\hline Age (years) & 34.0 & 14.5 & 4 & 78 \\
Track segment length (metres) & 7467 & 3451 & 722 & 17014 \\
Total traffic volume (gross tonnes) & 8644145 & 7179943 & 303406 & 27485512 \\
Total trains (number of) & 12740 & 8492 & 866 & 55831 \\
Freight traffic volume (gross tonnes) & 7139853 & 7026364 & 82064 & 26818684 \\
Freight trains (number of) & 5678 & 3943 & 185 & 11925 \\
Passenger traffic volume (gross tonnes) & 1504292 & 1865967 & 1308 & 14700000 \\
Passenger trains (number of) & 7061 & 8335 & 7 & 55364 \\
Dummy - Continuously Welded Rails & 0.687 & & 0 & 1 \\
Dummy - Concrete sleepers & 0.288 & & 0 & 1 \\
Dummy - Region North & 0.184 & & 0 & 1 \\
Dummy - Region East & 0.067 & & 0 & 1 \\
Dummy - Region West & 0.245 & & 0 & 1 \\
Dummy - Region South & 0.209 & & 0 & 1 \\
\hline
\end{tabular}

Note: Total traffic includes only freight and passenger traffic, other traffic such as maintenance traffic is not included. Traffic volume and number of trains is expressed per track, i.e. for double tracks the volume is divided by $2 . n=163$

Table 1 gives the basic descriptive statistics of the uncensored observations, i.e., track segments that have been replaced sometime between 1999 and 2009. Table 2 shows the corresponding statistics of the censored observations, i.e., track segments that are still "alive" in year 2009 . 
Table 2. Descriptive statistics of the censored observations in the data set

\begin{tabular}{lrrrr}
\hline Variable & Mean & Std. Dev. & Min & Max \\
\hline Age (years) & 24.8 & 10.5 & 12 & 66 \\
Track segment length (metres) & 7737 & 4419 & 176 & 28341 \\
Total traffic volume (gross tonnes) & 6795856 & 4581925 & 157840 & 18727860 \\
Total trains (number of) & 14841 & 10144 & 283 & 58962 \\
Freight traffic volume (gross tonnes) & 3953509 & 3835044 & 1477 & 17133280 \\
Freight trains (number of) & 3988 & 3381 & 2 & 13145 \\
Passenger traffic volume (gross tonnes) & 2842051 & 3169436 & 144 & 14652039 \\
Passenger trains (number of) & 10853 & 9846 & 1 & 58962 \\
Dummy - Continuously Welded Rails & 0.837 & & 0 & 1 \\
Dummy - Concrete sleepers & 0.782 & & 0 & 1 \\
Dummy - Region North & 0.095 & & 0 & 1 \\
Dummy - Region East & 0.288 & & 0 & 1 \\
Dummy - Region West & 0.189 & & 0 & 1 \\
Dummy - Region South & 0.219 & & 0 & 1 \\
\hline Note: Tatal trafic include & & & 0 & 0 \\
\hline
\end{tabular}

Note: Total traffic includes only freight and passenger traffic, other traffic such as maintenance traffic is not included. Traffic volume and number of trains is expressed per track, i.e. for double tracks the volume is divided by $2 . n=1109$

\section{Estimated survival models}

In this section, the results from different model specifications of the survival analysis are presented. The purpose of the survival analysis is to estimate models that provide input into expression (13), namely the deterioration elasticity $\varepsilon$, the scale and shape parameters $\lambda$ and $\alpha$ as well as the expected life time $\mu$.

The parametric survival model with a Weibull distribution has an accelerated failure time representation as well as a linear representation in the logarithm of time (or age) (Klein and Moeschberger, 2002). Let $x$ be the observed age of observation $i$ and $\mathbf{z}_{i}$ a vector of covariates fixed over time for the same observation. Cleves et al. (2004) formulates age in the AFT model as $x_{i}^{*}=\exp \left(-\mathbf{z}_{i}^{\prime} \boldsymbol{\beta}\right) x_{i}$ where $x_{i}^{*} \sim \operatorname{Weibull}(\lambda, \alpha)$. The intuition behind the AFT formulation is that $\exp \left(-\mathbf{z}_{i}^{\prime} \boldsymbol{\beta}\right)$ works as an acceleration factor on $x$. If the covariates in $\mathbf{z}$ are zero, $x_{i}=x_{i}^{*}$, which is the baseline risk. Every observation faces the same hazard function, but as time goes by, the acceleration factor generated by the individual covariates will affect the passage of time itself. The AFT representation can be rewritten as 
$\ln \left(x_{i}\right)=\mathbf{z}_{i}^{\prime} \boldsymbol{\beta}+\ln \left(x_{i}^{*}\right)=\beta_{0}+\mathbf{z}_{i}^{\prime} \boldsymbol{\beta}+u_{i}$

where $u_{i}$ is extreme value distributed with shape parameter $\alpha$.

Maximising the following log-likelihood function will give the maximum likelihood estimates of $\boldsymbol{\theta}=(\alpha, \boldsymbol{\beta}, \lambda)$ in the presence of right censored observations (Wooldridge, 2002).

$\ln L(\boldsymbol{\theta})=\sum_{i=1}^{N}\left\{d_{i} \ln \left[f\left(x_{i} \mid \mathbf{z}_{i} ; \boldsymbol{\theta}\right)\right]+\left(1-d_{i}\right) \ln \left[1-F\left(x_{i} \mid \mathbf{z}_{i} ; \boldsymbol{\theta}\right)\right]\right\}$

where $d_{i}=1$ for uncensored observations; 0 otherwise, $N=$ number of observations, $\mathbf{z}_{i}=\mathrm{a}$ vector of covariates for observation $i, \boldsymbol{\theta}=$ a vector of unknown model parameters to be estimated. $f()$ is the probability density function and $F()$ is the cumulative distribution function. The second term equals the logarithm of the survival function of the Weibull model and this is the only information that the censored observations provides, the probability of surviving at least to $x$. Conversely, the uncensored observations provide the unconditional probability of being renewed in time $x$.

Traffic volumes are expected to be the most important covariate for life time length of the track segments. Here, total tonnage per segment is compared to splitting the total into freight and passenger tonnage. All volumes are divided by the number of tracks per segment. A negative relationship between traffic volumes and life times is expected. We also expect that not only traffic will affect the renewal times, but more likely a combination of traffic and infrastructure characteristics. Effects from dummy variables for continuously welded rails, concrete sleepers, rails below $60 \mathrm{~kg}$ per metre, and single tracks are included in the models. Previous analyses of cost data for maintenance and renewal (Andersson, 2006) indicate that effects of track district location have a role in explaining cost variations over the Swedish 
railway network and therefore we also include dummy variables for track regions in the models. Finally, we estimate models with track region as frailty instead of regions as dummy variables. The statistical software package Stata, version 11 (StataCorp, 2009) is used for all model estimations.

We use three model specification tests. Firstly, a likelihood ratio test $(L R)$ is performed for the specified models versus a constant only model. Secondly, Akaike's (AIC) and Bayes Information Criteria $(B I C)$ are also used. These criteria penalise complex models by adding a factor to the traditional log likelihood calculation as the number of model parameters are increased. The general formula for these criteria is $I C=-2 \log (\hat{L})+\gamma K$ where $K$ is the number of free parameters in the model. The difference between the two versions is that the AIC uses $\gamma=2$ and BIC uses $\gamma=\log (N)$ where $N$ is the number of observations in the model estimation. The BIC puts a heavier penalty on the log likelihood than the AIC for large samples.

Finally, the last model specification test is to plot the Cox-Snell (pseudo) residuals against the cumulative hazard function $H(x)$ as recommended by Klein and Moeschberger (2002). In a correct model specification, the residuals follow a 45 degree reference line. Strong deviations from this line indicate a misspecified model.

All model coefficients are expressed in AFT format. The results of the estimated models are given in table 3 .

The dummy variables for single tracks and rail weight were both insignificant and were omitted from the models. Model 1 and Model 2 describe the reduced and re-estimated models.

The insignificant single track dummy means that all variation due to traffic loads distributed over several tracks are controlled for in our gross tonnes variables, which are divided by the number of tracks on each segment. The insignificant rail weight dummy indicates that the features of rails and sleepers are captured by the variables continuously 
welded rails and concrete sleepers. Most track region dummy variables turn out significant, indicating some regional differences.

Table 3. Weibull AFT models with traffic, infrastructure variables and track region dummies

\begin{tabular}{|c|c|c|c|c|}
\hline \multirow[t]{2}{*}{ Variable } & \multicolumn{2}{|c|}{ Model 1} & \multicolumn{2}{|c|}{ Model 2} \\
\hline & Coeff. & (S.E.) & Coeff. & (S.E.) \\
\hline Constant & 8.368 & $+(0.685)$ & 1.930 & $(1.886)$ \\
\hline $\ln$ Total Gross Tonnes & -0.302 & $+(0.049)$ & & - \\
\hline $\ln$ Freight Gross Tonnes & & - & 0.401 & $(0.259)$ \\
\hline$(\ln \text { Freight Gross Tonnes })^{2}$ & & - & -0.022 & $\dagger(0.010)$ \\
\hline InPassenger Gross Tonnes & & - & 0.144 & $*(0.081)$ \\
\hline$(\ln \text { Passenger Gross Tonnes })^{2}$ & & - & -0.006 & $*(0.004)$ \\
\hline D-Continuously Welded Rails & 0.321 & $\dagger(0.121)$ & 0.350 & $+(0.123)$ \\
\hline$D-$ Concrete sleepers & 0.156 & $\dagger(0.066)$ & 0.142 & $\dagger(0.063)$ \\
\hline$D-$ Region North & -0.192 & $+(0.060)$ & -0.185 & $\ddagger(0.058)$ \\
\hline$D-$ Region East & 0.156 & $*(0.090)$ & 0.037 & $(0.087)$ \\
\hline$D-$ Region West & -0.114 & $*(0.065)$ & -0.165 & $\dagger(0.068)$ \\
\hline D-Region South & 0.025 & $(0.070)$ & -0.089 & $(0.072)$ \\
\hline$\alpha$ & 3.976 & $(0.244)$ & 4.239 & $(0.264)$ \\
\hline Log likelihood & & -249.71 & & -243.29 \\
\hline Likelihood Ratio $\chi^{2}$ & & 134.31 & & 147.14 \\
\hline Number of observations & & 1272 & & 1272 \\
\hline $\mathrm{AIC}$ & & 517.42 & & 510.58 \\
\hline $\mathrm{BIC}$ & & 563.75 & & 572.36 \\
\hline
\end{tabular}

Since a regression model in logarithmic form is used, the coefficients can be interpreted as elasticities (Gujarati, 1995). The coefficient for total gross tonnes in Model 1 is the deterioration elasticity with a point estimate of -0.302 . A percentage increase in gross tonnes would lead to a 0.3 per cent reduction in expected rail life, ceteris paribus. Since Model 2 includes a squared term, the elasticity is not constant over the range of traffic as in Model 1. Expression (16) is used to calculate track segment specific deterioration elasticities, $\hat{\varepsilon}_{i}$, of Model 2.

$\hat{\varepsilon}_{i}=\frac{\partial \ln X}{\partial \ln q_{i}}=\hat{\beta}_{\ln q}+2 \hat{\beta}_{(\ln q)^{2}} \ln q_{i}$ 
The mean of these elasticities, $\bar{\varepsilon}$ (standard error), are for freight traffic $-0.236(0.036)$ and for passenger traffic $-0.034(0.028)$. Hence, a percentage increase in passenger traffic reduces rail life on average by 0.03 per cent while the reduction from freight is 0.24 per cent. However, the elasticity for passenger traffic is not significantly different from zero, even at the ten per cent level.

The Weibull shape parameter $(\alpha)$ is close to 4 in both Model 1 and Model 2 indicating strong, positive duration dependence (increasing risk over time) as well as a more than proportional acceleration of the renewal risk with respect to accumulated traffic.

The LR test favours both models before a constant only model. Furthermore, the BIC is in favour of Model 1 whereas the AIC favours Model 2. Plotting the Cox-Snell residuals for both models indicate a better fit though for Model 1 than for Model 2, see Figure 3.
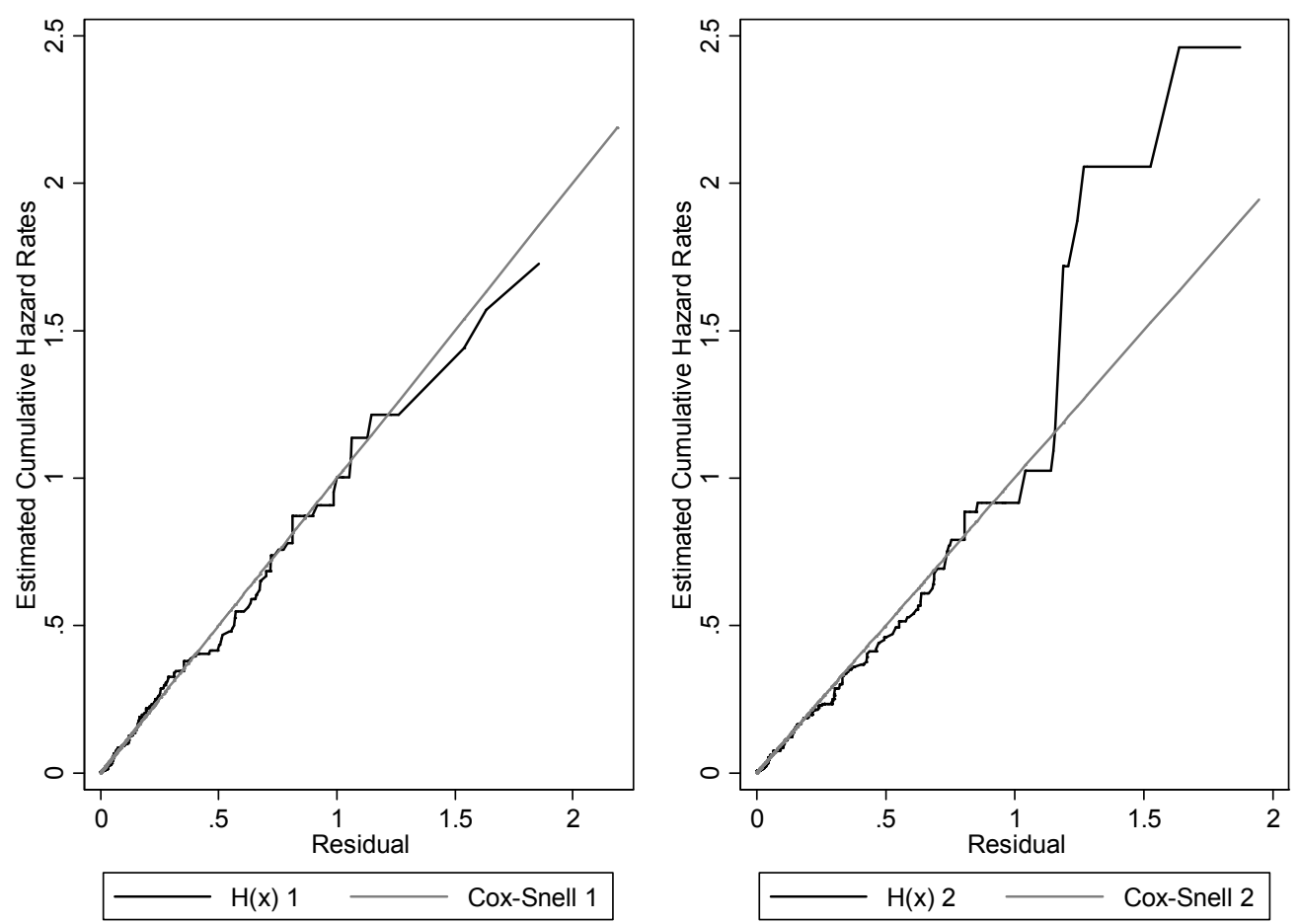

Figure 3. Cox-Snell (C-S) residual specification test for Model 1 and 2 
We also estimated Model 1 and Model 2 with region as a shared variable in frailty models, where the shared variable, or frailty, is an unobservable random effect shared by segments in a region. However, this resulted in a worse fit for both models and we did not go further with the frailty estimations. Our preferred model therefore is Model 1 according to the specification tests and further analyses are based on this model, i.e. the model with total tonnage. We also tested a squared term for the traffic volume in Model 1, but this did not improve the model. The hazard and survival functions of Model 1 are presented in Figure 4. The survival probability is quite high for the first 25 years, but is then reduced in an accelerating manner. Unless maintenance activities fully compensate for wear, tear and climate effects, this pattern is expected, which is also shown as increasing hazard rates over time.
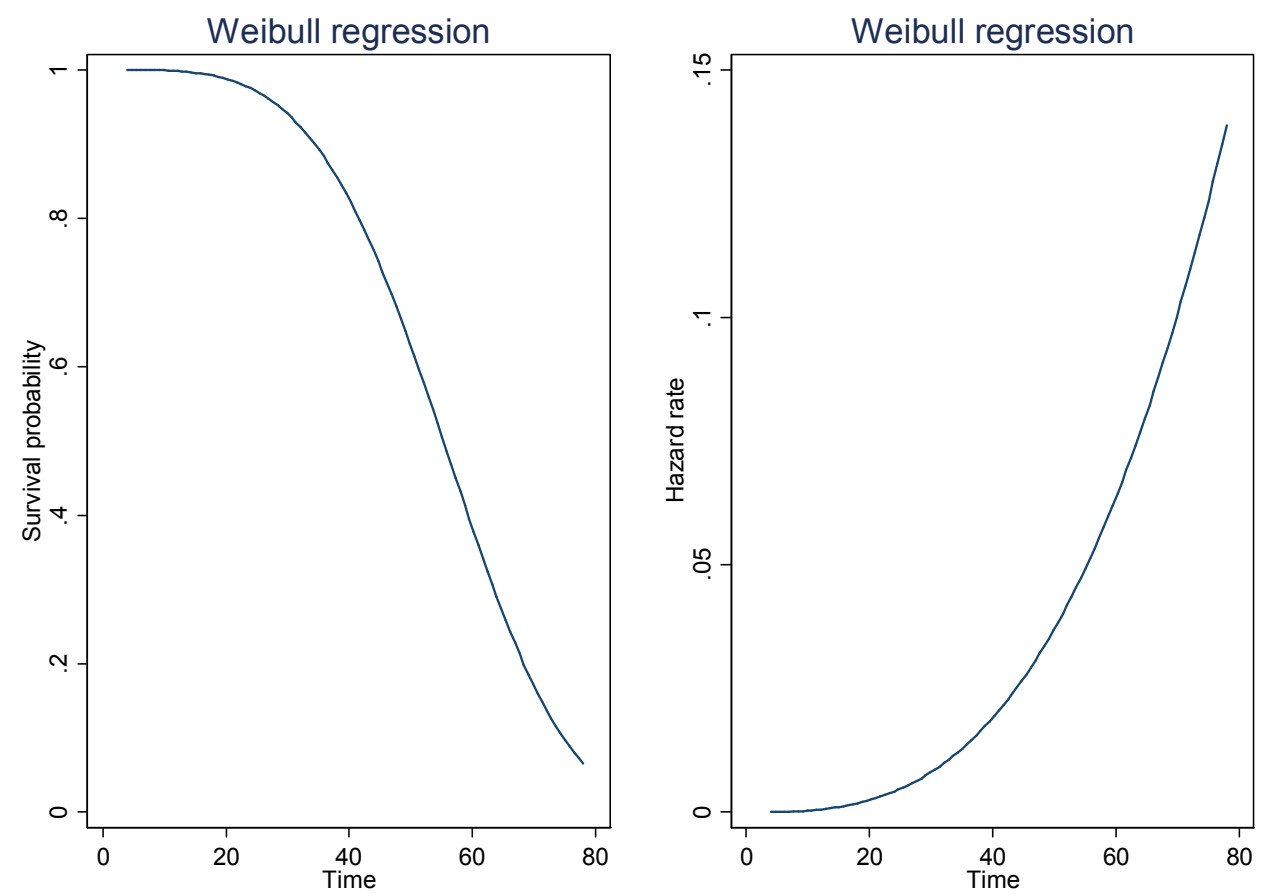

Figure 4. The survival and hazard functions for Model 1 


\section{Marginal costs}

Expression (17) follows (13) and is used for the marginal cost calculation. It contains four distinct parts. The first part is the deterioration elasticity, $\hat{\varepsilon}$, which is estimated in our survival model to be -0.302 . The second part, $\frac{c}{\bar{q}_{1} \hat{\mu}_{i}}$, is the average cost of a rail renewal. The third part, $\frac{r}{\left(1-e^{-r \bar{X}}\right)}$, is the discount factor of an infinite cycle of estimated average life times $\bar{X}$. The fourth part, the integral, adjusts the calculation to the distribution of rail ages and remaining expected life times in our sample. This part has no closed form and is solved by numerical integration. We limit the integration area to $0-100$, as the oldest rail segment in our sample is 78 years old.

$$
E\left[\frac{\partial P V C_{\widetilde{x}}}{\partial q_{\widetilde{x}}}\right]=-\hat{\varepsilon} \frac{c}{\bar{q}_{1} \hat{\mu}_{i}} \frac{r}{\left(1-e^{-r \bar{x}}\right)} \int_{0}^{\infty} e^{-r \omega-\hat{\lambda}_{i} \omega^{\hat{\alpha}}} d \widetilde{x}
$$

$c$ is the track renewal cost and is estimated by taking the sum of all renewal costs between 1999 and 2009 for track segments included in the sample and dividing this sum by the sum of all track lengths that have been replaced during the same period. This operation resulted in an average cost of approximately SEK 2.6 million (2009 prices) per kilometre track. The cost data originate from Trafikverket's accounting system, Agresso. $r$ is the social discount rate, which in Swedish transport infrastructure projects is set to 4 per cent. The predicted life time for an observation, is calculated as $\hat{\mu}_{i}=\exp \left(\mathbf{z}_{i}^{\prime} \boldsymbol{\beta}\right) . \hat{\alpha}$ is the shape parameter for the Weibull distribution, estimated to 3.976 for Model 1. The scale parameter $\hat{\lambda}_{i}$ is observation specific and is calculated as $\hat{\lambda}_{i}\left(x \mid \mathbf{z}_{i}\right)=\hat{\mu}_{i}^{-\hat{\alpha}}$. The calculations give an average marginal cost estimate of SEK 0.0052 (0.0002) per gross tonne kilometre. 
However, using a simple mean value in a pricing scheme, would place too much weight on observations with low traffic volumes. In previous studies, Andersson $(2006,2008)$ handles this by placing different weights on the track segment specific marginal costs in accordance with the segments share of total gross tonne kilometres. This is a revenue-neutral scaling procedure, and gives a weighted average marginal cost of SEK $0.0021(0.0003)$ per gross tonne kilometre in the present study. Applying this estimate to all segments gives the same total revenue as using segment individual estimates.

\section{Discussion and conclusions}

Pricing at marginal cost for railway use is important from an efficiency standpoint. In this paper, we have studied the overall issue of railway track wear and tear, and specifically the issue of marginal costs related to rail renewal. In contrast to previous cost function efforts, a method that calculates the difference in the present value of rail track renewal costs related to changes in rail renewal cycles from different levels of traffic is used. A crucial factor in this calculation is to estimate the deterioration elasticity, the percentage change in rail life from a percentage change in traffic. This is done using survival analysis in the form of an accelerated failure time Weibull model. Several specifications of the Weibull model are analysed and we test for potential errors in the specifications for two of the models. A model that is based on total tonnage (the sum of freight and passenger tonnage) is recommended. We estimate a weighted average marginal cost to approximately SEK 0.002 per gross tonne kilometre.

There are some points that should be emphasised. The first is that these estimates are similar to previous econometric cost function estimates. Andersson (2006) applies pooled ordinary least squares (POLS) to track section cost data and finds that the increase in weighted marginal cost, when renewals are added to maintenance costs, is SEK 0.0024 per gross tonne kilometre. Andersson et al. (2012) estimates the weighted marginal cost to SEK 
0.007 per gross tonne kilometre. One has to be aware of the different cost bases used in this study, Andersson (2006), and Andersson et al. (2012) respectively. In this study and in the study by Andersson et al. (2012), track renewal costs are used, while Andersson (2006) use all renewal costs. Although this study and Andersson et al. (2012) use the same cost base, the precision in using an average cost estimate as we do is not as good as observation specific costs used in Andersson et al. (2012). In that study it was possible to use track section cost data. Such data is not available for track segments used in the present study. The cost representation is therefore something to focus on in future research.

Secondly, there seems to be strong evidence for a price relevant rail track renewal cost in Sweden, which should be included in future pricing schemes if marginal cost pricing is aimed at. The size of such a wear and tear fee for rail renewal depends on how the actual pricing scheme is designed, either using a flat rate for all track segments or segment specific estimates. The latter is a more complicated approach as it in theory requires an iterative process between time-tabling and deciding on optimal access charges. Our proposal is therefore a network weighted average charge around SEK 0.002 per gross tonne kilometre. The latest Network Statement by Trafikverket (Trafikverket, 2011) holds the official wear and tear charge for 2012, which is SEK 0.0036 per gross tonne kilometre. The latest revision of this charge is based on econometric analyses of maintenance costs in Andersson (2006). Charging for track renewals as well would increase the amount payable by Swedish train operators by some fifty per cent for infrastructure wear and tear. Furthermore, Andersson (2008) has re-estimated the models in Andersson (2006) using fixed effects models. This has resulted in a marginal cost estimate in the range of SEK 0.007 per gross tonne kilometre indicating that railway infrastructure wear and tear charges in Sweden today are well below marginal cost. 
The third point is that the method as such is working, which can be an opening for marginal cost estimates when long time-series of renewal cost data are not at hand. There are two main sources of information that are necessary in this case. One is a well-functioning track information system and the other is a traffic information system. It is important to point out that this information is needed for a few years only, during which at least some renewals have been undertaken. Modern railway track information systems have the possibility to record not only the current status of the network at a detailed level, but also its previous status. This will, over time, generate a rich information source for this type of analysis and adding more uncensored observations to the database, will hopefully increase precision in our estimates.

An obvious field of development is to make a good representation of the infrastructure variables. We have not been able to identify different types of tracks being of importance for our estimates. One reason for this can be that the underlying spending pattern on maintenance during the life cycle is adjusted in such a way that the inherent quality differences between tracks are levelled out. This is an area where more work is needed in the future, and where an extended database will provide possibilities for some answers.

The marginal cost is highly dependent on and directly proportional to the cost estimate for rail track renewals calculated in this study. To get a better estimate, we need to look closer at rail renewals of different types to be able to predict the rail renewal cost at the track segment level. More specific costs for different track types would improve this part.

On the theoretical representation, introducing a stochastic representation of future renewal intervals could be the next area to develop. We are currently assuming all future intervals being equal, which most likely is too strong. Whether this would have an effect on the marginal cost estimates is difficult to say, but since we use a discounting procedure, future costs are of less importance and we do not expect any dramatic effects. 


\section{Acknowledgments}

Financial support from Trafikverket and the European Commission is gratefully acknowledged. This paper has benefited from comments by Lars-Göran Mattsson, Jan-Eric Nilsson, Matias Eklöf, Kenneth Small and an anonymous referee. Special thanks to Mattias Haraldsson for valuable model discussions and marginal cost calculations. The authors take full responsibility for any remaining errors.

\section{References}

Andersson, M. (2006) Marginal Cost Pricing of Railway Infrastructure Operation, Maintenance and Renewal in Sweden: From Policy to Practice Through Existing Data. Transportation Research Record: Journal of the Transportation Research Board, No. 1943, Transportation Research Board of the National Academies, Washington, D.C., pp. 1-11.

Andersson, M. (2008) Marginal railway infrastructure costs in a dynamic context. European Journal of Transport Infrastructure Research, 8(4), 268-286.

Andersson, M. (2011) Marginal cost of railway infrastructure wear and tear for freight and passenger trains in Sweden. European Transport, 40, 3-23.

Andersson, M., Smith, A., Wikberg, Å., and Wheat, P. (2012) Estimating the marginal cost of railway track renewals using corner solution models. Transportation Research A, 46, 954964.

Bruzelius, N. (2004) Measuring the Marginal Cost of Road Use - An International Survey. VTI Meddelande 963A. Linköping, Sweden. 
Cleves, M.A., Gould, W.W. and Gutierrez, R.G. (2004) An Introduction to Survival Analysis Using Stata. Stata Press, College Station, Tx.

Gujarati, D.N. (1995) Basic Econometrics. 3rd ed., McGraw-Hill, Singapore.

Haraldsson, M. (2007) Essays on transport economics. Ph.D. Thesis, Department of Economics, Uppsala University, Sweden.

Johansson, P. and Nilsson, J.-E. (2004) An economic analysis of track maintenance costs. Transport Policy, 11(3), 277-286.

Kiefer, N.M. (1988) Economic duration data and hazard functions. Journal of Economic Literature, 26(2), 646-679.

Klein, J.P. and Moeschberger, M.L. (2002) Survival Analysis - Techniques for Censored and Truncated Data. 2nd ed. Springer, New York, NY.

Lancaster, T. (1990) Econometric Analysis of Transition Data. Cambridge University Press, Cambridge, UK.

Lindberg, G. (2002) Marginal Cost of Road Maintenance for Heavy Goods Vehicles on Swedish Roads. Annex A2, Deliverable 10: Case Studies on Marginal Infrastructure Costs, UNITE (UNIfication of accounts and marginal costs for Transport Efficiency), Funded by 5th Framework RTD Programme, Institute for Transport Studies, University of Leeds, Leeds, UK. 
Link, H. and Nilsson, J.-E. (2005) Infrastructure. Measuring the Marginal Social Cost of

Transport eds. C. Nash and B. Matthews. Research in Transportation Economics, Vol. 14, pp. 49-83. Elsevier, Oxford, UK.

Nash, C. (2003) UNITE (UNIfication of accounts and marginal costs for Transport Efficiency). Final Report for Publication. Funded by 5th Framework RTD Programme, Institute for Transport Studies, University of Leeds, Leeds, UK.

Nash, C. (2005) Rail infrastructure charges in Europe. Journal of Transport Economics and Policy, 39(3), 259-278.

Nash, C. and B. Matthews (Eds.) (2005) Measuring the Marginal Social Cost of Transport, Research in Transportation Economics, Vol. 14. Elsevier, Oxford, UK.

Nash, C. and Sansom, T. (2001) Pricing European transport systems: Recent developments and evidence from case studies. Journal of Transport Economics and Policy, 35(3), 363-380.

Newbery, D.M. (1988) Road user charges in Britain. The Economic Journal, 98(390), 161176.

StataCorp. (2009) Stata Statistical Software: Release 11. College Station, Tx.

Thomas, J., Dionori, F. and Foster, A. (2003). EU task force on rail infrastructure charging. Summary findings on best practice in marginal cost pricing. European Journal of Transport and Infrastructure Research, 3(4), 415-431. 
Trafikverket (2011) Network Statement. 2011-12-11 - 2012-12-08(T12), Trafikverket, Borlänge, Sweden.

Wheat, P. and Smith, A. (2008) Assessing the marginal infrastructure maintenance wear and tear costs for Britain's railway network. Journal of Transport Economics and Policy, 42(2), $189-224$.

Wooldridge, J.M. (2002) Econometric Analysis of Cross Section and Panel Data. MIT Press, Cambridge, Mass. 